\title{
To Study the Utilization Pattern of Nasal Decongestants and their Effects on Heart Rate and Blood Pressure
}

\author{
Harmeet Singh Rehan, Asmita Chopra, Sunil Kumar
}

\begin{abstract}
Background: Nasal decongestants (NDs) are frequently prescribed over the counter (OTC) drugs for cough and cold. These are sympathomimetic drugs, which act through $\alpha_{1}$ and $\beta_{1}$ adrenoceptors to cause vasoconstriction and positive inotropic, chronotropic and dromotropic effect on the heart. This study was conducted to assess the pattern of utilization of the NDs and their effect on heart rate (HR) and blood pressure (BP). Patients were observed for any other adverse drug reactions (ADRs).

Materials and methods: Randomly 100 prescriptions containing NDs were collected from the otorhinolaryngology OPD and were analyzed. All these patients were examined for HR and BP on day 0,3 and 7 of administration of NDs. Any ADRs were also recorded.
\end{abstract}

Results: Mean ND used per patient was 1.08/patient. Commonly prescribed NDs were phenylephrine $(72.2 \%)$, pseudoephedrine (12.9\%), phenylpropanolamine (6.5\%), xylometazoline $(6.5 \%)$ and oxymetazoline (1.8\%). Eight patients received two NDs orally and/or topically. There was a significant rise in heart rate on day $7(p<0.05)$. However, the rise in BP was insignificant both on days 3 and 7 . Frequently observed ADR's included headache, palpitations and dizziness.

Discussion: Some NDs are known to raise the HR and BP. Baseline BP monitoring should be done to avoid any further rise in BP and HR. being an OTC drug there are more chances of adverse effects of these NDs. It is suggested that NDs should be prescription only drugs, till adequate evidence is available regarding their safety.

Keywords: Nasal decongestants, Xylometazoline, Pseudoephedrine, Allergic rhinitis.

How to cite this article: Rehan HS, Chopra A, Kumar S. To Study the Utilization Pattern of Nasal Decongestants and their Effects on Heart Rate and Blood Pressure. Clin Rhinol An Int J 2012;5(3):91-94.

Source of support: Nil

Conflict of interest: None declared

\section{INTRODUCTION}

Common cold is a frequent and recurrent cause of morbidity in both adults and children. It commonly manifests with nasal congestion, which responds to orally or topically (drops or sprays) administered nasal decongestants (NDs). The commonly used NDs are phenylephrine, pseudoephedrine, phenylpropanolamine, oxymetazoline, xylometazoline, naphazoline, etc.

Oral NDs take approximately 15 to 30 minutes to act, while the topical ones act much faster. Most of the NDs stimulate $\alpha_{1}$ adrenergic receptors, to produce vasoconstriction of the blood vessels in the nasal mucosa, thus reducing its swelling and congestion.
The pharmacological effects of NDs are not limited to the nose, but produce systemic effects also. The orally administered NDs produce more systemic side effects than the topically administered ones. Side effects include their pharmacological effects on the cardiovascular system through $\alpha_{1}$ adrenergic agonist action which produces vasoconstriction and increases peripheral vascular resistance and $\beta_{1}$ adrenergic agonistic action that has a positive inotropic effect on the heart. ${ }^{1}$

In 2000, Kernan et al, reported that the anorectic use of the ND, phenylpropanolamine, is associated with CVA. ${ }^{2}$ In view of such reports and the observation of Hemorrhagic Stroke Project the FDA banned the use of phenylpropanolamine in the year $2000 .{ }^{3}$ However, the safety status of phenylpropanolamine and other NDs in India is not yet clear due to the lack of evidence.

Hence, the present study was planned to generate baseline data on the pattern and safety of use of NDs including their fixed dose combinations and to evaluate the effect of NDs on the heart rate (HR) and blood pressure (BP).

\section{MATERIALS AND METHODS}

An observational study was conducted to analyze the prescriptions written by otorhinolaryngologists containing NDs. Prescriptions were randomly collected outside the OPD and analyzed for indications for which NDs were prescribed; concurrently prescribed groups of medicines; prescription by generic or brand name; type and route of administration of NDs. Drugs in the fixed dose combinations (FDCs) were considered as a single drug. Study protocol was approved by the Institutional Ethical Committee.

All these naive 100 patients, prescribed NDs or fixed dose combinations containing ND and agreed to participate in the study, were examined for HR and BP on days 0,3 and 7. Observed adverse drug reactions (ADRs) recorded and analyzed for causality using the Naranjo probability scale. ${ }^{4}$ Any change in HR and BP after 3 and 7 days of the use of NDs was compared with the baseline, i.e. day 0 data using Student's t-test. A p $<0.05$ was considered statistically significant.

\section{RESULTS}

All the 100 patients (52 females and 48 males) whose prescriptions were collected for analysis participated and 
completed the study. Mean age of the study patients was $33.57 \pm 1.45$ years (range, $12-70$ years).

Hundred patients were diagnosed with 117 conditions, 14 patients had more than one diagnosis. Commonest indication for the use of nasal decongestion was rhinitis (23.9\%), followed by chronic suppurative otitis media (17.9\%) and tympanic membrane retraction and congestion (14.5\%; Table 1). All the medicines in this study were prescribed by brand names. A total of 347 medicines were prescribed with an average of 3.47 medicines per patient. FDCs were frequently (67\%) used formulations (Table 2).

Commonly prescribed group of drugs along with NDs (31.1\%) were antihistaminics (29\%) and NSAIDs (25.9\%; Table 3). A total of 108 ND containing drugs were prescribed to 100 patients, with an average of 1.08 NDs per patient. All the oral NDs prescribed were FDCs with antihistamines and/or NSAIDs (Table 2). Topically used NDs were single drug preparations containing either

\begin{tabular}{lc}
\multicolumn{2}{c}{$\begin{array}{c}\text { Table 1: Diagnosis of the } \mathbf{1 0 0} \text { patients receiving NDs } \\
\text { included in the study (N = 117) }\end{array}$} \\
Diagnosis & No. of diagnosis \\
\hline Aural & 55 \\
CSOM & 19 \\
Otomycosis & 05 \\
TM retraction & 17 \\
Pain and decreased hearing & 14 \\
Nasal & 44 \\
Rhinitis & 28 \\
Nasal blockage & 08 \\
Epistaxis & 05 \\
Sinusitis & 02 \\
DNS & 01 \\
Throat & 18 \\
Cough & 06 \\
Pain and irritation & 05 \\
Others & 07
\end{tabular}

Table 2: Type of fixed dose combinations prescribed $(\mathrm{N}=128)$

\begin{tabular}{ll} 
Fixed dose combinations & No. (\%) \\
\hline Phenylephrine + chlorpheniramine maleate (CPM) & $39(30.4)$ \\
Phenylephrine + CPM + paracetamol (PCM) & $33(25.7)$ \\
Pseudoephedrine + levocetirizine & $13(10.1)$ \\
Clotrimoxazole + chloramphenicol & $08(06.2)$ \\
Phenylephrine + ebastine & $07(05.4)$ \\
Phenylpropanolamine + PCM + CPM & $07(05.4)$ \\
PCM + ibuprofen & $05(03.9)$ \\
Other NSAIDs combinations & $07(05.4)$ \\
Others & $09(07.0)$
\end{tabular}

Table 3: Frequently prescribed groups of drugs $(\mathrm{N}=347)$

\begin{tabular}{lr} 
Groups of drugs & No. (\%) \\
\hline Nasal decongestants & $108(31.1)$ \\
Antihistamines & $101(29.1)$ \\
NSAIDs & $90(25.9)$ \\
Antibiotics & $26(07.5)$ \\
Other & $22(06.3)$ \\
\hline
\end{tabular}

oxymetazoline or xylometazoline. Eight patients received two NDs concurrently. Majority of the patients received single oral decongestant (83.3\%) and one patient received two oral FDCs both of which contained an ND each, i.e. pseudoephedrine and phenylpropanolamine. Seven patients received oral and topical NDs concurrently. Two patients received single topical ND. Among the NDs prescribed phenylephrine was the most frequently prescribed (78\%), followed by pseudoephedrine (Table 4).

\section{Table 4: Type and frequency of the use of NDs ( $N=108)$}

\begin{tabular}{lc} 
Nasal decongestants & No. (\%) of prescriptions* \\
\hline Phenylephrine & $78(72.2)$ \\
Pseudoephedrine & $14(12.9)$ \\
Phenylpropanolamine & $07(6.5)$ \\
Oxymetazoline & $07(6.5)$ \\
Xylometazoline & $02(1.8)$ \\
\hline
\end{tabular}

*Eight patients received more than one NDs

Mean baseline HR was $80.79 \pm 1.07 /$ min (range, 56$102 / \mathrm{min}$ ). It increased to $82.74 \pm 1.01 / \mathrm{min}$ (range, 63-103/ $\mathrm{min}$ ) and to $84.33 \pm 1.05 / \mathrm{min}$ (range, $63-105 / \mathrm{min}$ ) on days 3 and 7 respectively.

Mean baseline systolic BP was $117 \pm 1.09 \mathrm{~mm} \mathrm{Hg}$ (range, 92-136 mm Hg). It increased to $119.28 \pm 1.12 \mathrm{~mm} \mathrm{Hg}$ (range, 96-146 mm Hg) and then to $119.92 \pm 1.137$ (range, 100-152 mm Hg) on days 3 and 7 respectively.

Mean baseline diastolic BP was $76.16 \pm 0.98 \mathrm{~mm} \mathrm{Hg}$ (range, $52-78 \mathrm{~mm} \mathrm{Hg}$ ). It increased to $76.88 \pm 0.95 \mathrm{~mm} \mathrm{Hg}$ (range, 54-96 $\mathrm{mm} \mathrm{Hg}$ ) and then to $77.52 \pm 0.97 \mathrm{~mm} \mathrm{Hg}$ (range, 58-100 mm Hg) on days 3 and 7 respectively (Table 5).

A total of 41 ADRs including headache (17), palpitations (11), dizziness (5), nasal bleeding (2) and others (6) were observed. Causality assessment was probable for 22 and possible for 19 ADRs. Patients who reported increased nasal bleeding following NDs, subsided itself despite continuation of NDs.

\section{DISCUSSION}

Polypharmacy increases the incidence of ADRs and drug interactions. ${ }^{5}$ In this study mean number of drugs per prescription was 3.47. The mean drug per patient was high (3.47) in this study. Similarly polypharmacy (3.3-5.7 drugs/ patient) has been reported from Nepal, Nigeria, Indonesia and India. ${ }^{5-9}$

All the drugs (100\%) prescribed including NDs were prescribed by proprietary name and this was higher than that reported (70.7\%) by Rehan et $\mathrm{al}^{6}$ from Nepal, in patients from all the OPDs. Generic drugs are always costless and have similar efficacy as the drugs with proprietary names. Despite having hospital drug formulary and generic 
To Study the Utilization Pattern of Nasal Decongestants and their Effects on Heart Rate and Blood Pressure

\begin{tabular}{lccc}
\hline & \multicolumn{3}{c}{ Table 5: Effect of NDs on systolic and diastolic BP and HR (mean \pm SEM) } \\
Duration & $H R$ (beats/min) & Systolic BP $(\mathrm{mm} \mathrm{Hg})$ & Diastolic BP $(\mathrm{mm} \mathrm{Hg})$ \\
\hline Day 0 (baseline) & $80.79 \pm 1.07$ & $117 \pm 1.09$ & $76.16 \pm 0.98$ \\
Day 3 & $82.74 \pm 1.01$ & $119.28 \pm 1.12$ & $76.88 \pm 0.95$ \\
Day 7 & $84.33 \pm 1.05^{\star}$ & $119.92 \pm 1.137$ & $77.52 \pm 0.97$ \\
\hline
\end{tabular}

${ }^{*} \mathrm{p}<0.05$

prescribing policy in this institution use of branded preparations was high. Prescribers need to be appraised with benefits of generic prescribing.

In this study, $67 \%$ of total drugs prescribed were FDCs (91.6\%; Table 2). None of the FDC's used in the study was recommended by the WHO. ${ }^{10}$ Higher use of FDCs has been reported by drug utilization studies from both developing ${ }^{9}$ and developed countries. ${ }^{11}$ On the contrary, in Nepal, Rehan et al reported lower use (5.4\%) of FDCs. Also, no study was available to compare the frequency of use of FDCs containing NDs. FDCs have more disadvantages than benefits. The patient may not actually need all the drugs in a combination. The patient is subjected additional side effects and expense. Also, the freedom to modify the dose of one ingredient in the combination is lost. ${ }^{12}$

Frequently prescribed NDs were phenylephrine (78\%), pseudoephedrine (13\%) and phenylpropanolamine (7\%; Table 2). Though the prescriptions containing NDs were only included in the study, but it was observe that eight patients received two NDs, one received both orally, whereas others received by oral and topical route. Since these drugs are sympathomimetic agents, use of more than one NDs may put the patient at risk of ADRs. There is no study available to compare these findings.

NDs are sympathomimetic drugs that stimulate $\alpha$ and $\beta$ adrenoceptors. The stimulation of $\alpha_{1}$ receptors on the smooth muscles in the blood vessels cause vasoconstriction leading to decongestion of the nasal mucosa. But along with $\beta_{1}$ receptors stimulation, they cause rise in HR and BP. ${ }^{1}$

This study reported a significant rise in the HR on day 7 of administration of NDs. There was an insignificant rise in the systolic and diastolic BP on both days 3 and 7 of administration of NDs. Westerveld et al have also reported that NDs have dose-dependent inhibitory action on total iNOS activity which appears to be beneficial because inducible NO synthase activity may exacerbate the inflammatory process. ${ }^{13}$ They also reported that NDs in vitro appear to have additional benefit in the treatment of upper respiratory tract infection.

Overall effect of all the NDs caused a significant rise in HR on day 7 but systolic and diastolic BP showed slight insignificant rise. Similarly, Hatton et al have reported in a meta-analysis, that phenylephrine causes a rise in HR and $\mathrm{BP}$, which is insignificant in a normotensive individual. ${ }^{14}$
The reason for the significant rise in HR in our study could be because of the fact that eight patients received two NDs simultaneously by same or different routes. Use of more than one ND may not provide additional benefit, as they act through the same receptor $\left(\alpha_{1}\right)$, although their adverse effects are added up. Therefore, it is appropriate to use a single ND if required.

Empey et al have reported a rise in pulse and systolic BP with pseudoephedrine at 120 and $180 \mathrm{mg}$ but not with less than 60 mg. ${ }^{15}$ Similar to our study, they did not find any rise in diastolic BP. In our study $30 \mathrm{mg}$ twice daily dose of pseudoephedrine did not cause any significant rise in systolic/diastolic BP. Pseudoephedrine in the dose of 60 mg or less may be optimal to achieve maximum nasal decongestion without cardiovascular and other unwanted effects. ${ }^{15}$ In another study, Britton et al reported that pseudoephedrine is effective in preventing reflex mucoid congestion after histamine challenge. ${ }^{16}$

In our study, PPA was the third commonly prescribed ND (Table 4). PPA is sympathomimetic amine present in many cough and cold preparations. PPA is also being abused as an anorectic agent. Many case reports and studies have discussed the occurrence of intracranial hemorrhage/stroke after PPA and other NDs. ${ }^{2,17,18}$ In a Hemorrhagic Stroke Project, Horwitz et al concluded that the use of PPA increased the risk of hemorrhagic stroke which subsequently led to its ban by FDA as a ND and anorectic agent. ${ }^{3}$

In the present study, HR was significantly increased after 7 days use of NDs whereas insignificant rise in BP, suggesting their safety with regard to rise in blood pressure on short term ( $<7$ days) topical administration of NDs. Systemic review of prospective studies on BP by John and Frank concluded that safety margin of PPA may decrease in subjects with patients with elevated basal sympathetic tone, e.g. overweight patients and patients with slightly elevated arterial BP. ${ }^{19}$

Structure of pseudoephedrine resembling amphetamines due to which they have similar CNS effects, i.e. wakefulness, alertness and decrease of sense of fatigue and adverse effects, i.e. headache, dizziness palpitation, etc. ${ }^{1}$ In our study, headache, palpitations and dizziness was commonly observed ADRs, which could be due to the above reason.

In India, no well-planned study is available to establish the safety of PPA and other NDs. Since NDs are available 
as over the counter drugs and have a potential to cause cardiovascular and other adverse effects, hence it is imperative to monitor patient's baseline HR and BP and rule out any underlying cardiovascular disease before prescribing these drugs. In view of lack of safety data of NDs in India, it is also suggested that NDs should be available as prescription only drugs till supporting safety data is available.

\section{REFERENCES}

1. Westfall TC, Westfall DP. Adrenergic agonists and antagonists in Goodman and Gilman's the pharmacological basis of therapeutics (11th ed). In: Brunton LL, Lazo JS, Parker KL (Eds). McGraw-Hill Medical Publishing Division, New York 2006;237-95.

2. Kernan W, Viscoli C, Brass L, et al. Phenylpropanolamine and the risk of hemorrhagic stroke. N Engl J Med 2000;343:1826-32.

3. Horwitz RI, Ralph I, Harold H, et al. Hemorrhagic Stroke Project. Food and Drug Administration 2000.

4. Naranjo CA, Busto U, Sellers EM, et al. A method for estimating the probability of adverse drug reactions. Clin Pharmacol Ther 1981;30:239-45.

5. Jyrkkj J, Enlung H, Korhonen MJ, et al. Pattern of drug use and factors associated with polypharmacy and excessive polypharmacy in elderly persons; Results of Kuopio 75+ study: A cross section analysis. Drugs Aging 2009;26(6):493-503.

6. Rehan HS, Nagarani MA, Rehan M. A study on the drug prescribing pattern and use of antimicrobial agents at a tertiary care teaching hospital in Eastern Nepal. Ind J Pharmacol 1998;30(3):175-80.

7. Hogerzeil HV, Bimo, Ross-Degnan D, Laing RO, Ofori-Adjei D, Santoso B, Azad Chowdhury AK, et al. Field tests for rational drug use in twelve developing countries. Lancet 1993;342:1408-10.

8. Patrick OE, Olumide GO, Augustine OO. Prescribing practices in two health care facilities in Warri, South Nigeria: A comparative study. Trop J Pharm Res 2003;2(1):175-82.

9. Rehan HS, Singh C, Tripathi CD, Kela AK. Study of drug utilization pattern in dental OPD at tertiary care teaching hospital. IJDR 2001;12:51-56.
10. World Health Organization. Essential drugs: WHO Model List, WHO Drug Information, (15th ed). WHO: 2007.

11. Hogerzeil HV. Promoting rational prescribing: An international perspective. Br J Clin Pharma 1995;39(1):1-6.

12. Patel V, Vaidya R, Naik D, et al. Irrational drug use in India: A prescription survey from Goa. J Postgrad Med 2005,51(1);9-12.

13. Westerveld G, Voss H, VanDerHee R, et al. Inhibition of NOS by nasal decongestants. Eur Resp J 2000;16:437-44.

14. Hatton RC, Winterstein AG, McKelvey RP, et al. Efficacy and safety of oral phenylepherine: Systematic review and metaanalysis. Ann Phamacother 2007;41(3):381-90.

15. Empey DW, Young GA, Letley E, John GC, Smith P, McDonnell KA, et al. Dose response study of nasal decongestant and cardiovascular effects of pseudoephedrine. Br J Clin Pharmac 1980;9(4):351-58.

16. Britton MG, Empey DW, Johan GC, McDonnell KA, Huggies DTD. Histamine challenge and anterior nasal rhinometry: Use in assessment of pseudoephedrine. Br J Clin Pharmac 1978;6:51-58.

17. Prasad A, Bhoi K, Bala K, et al. Phenylpropanolamine induced intraventricular hemorrhage. Neurol India 2003 Mar;51(1): 117-18.

18. Cantu C, Arauz A, Murillo-Bonilla LM, et al. Stroke associated with sympathomimetics contained in OTC cough and cold preparations. Stroke 2003;34:1667-72.

19. Morgan JP, Funderburk FR. Phenylpropanolamine and blood pressure. Am J Clin Nutr 1992;55:206s-10s.

\section{ABOUT THE AUTHORS}

\section{Harmeet Singh Rehan (Corresponding Author)}

Professor and Head, Department of Pharmacology, Lady Hardinge Medical College and SSK Hospital, New Delhi, India, e-mail: harmeetrehan@hotmail.com

\section{Asmita Chopra}

Intern, Lady Hardinge Medical College and SSK Hospital, New Delhi India

\section{Sunil Kumar}

Professor, Department of ENT and Otorhinolaryngology, Lady Hardinge Medical College and SSK Hospital, New Delhi, India 